\title{
Numerical Simulation of Water Entry Impact of Torpedo Based on FE-SPH Method
}

\author{
PAN Cheng Wei ${ }^{1, a^{*}}$, HUANG Qiao Gao ${ }^{2, b}$ \\ ${ }^{1}$ The Middle School Attached To Northwestern Polytechnical University, Xi'an, China, 710072 \\ ${ }^{2}$ College of Marine, Northwestern Polytechnical University, Xi'an, China, 710072 \\ apancw_nwpu@163.com, bhuangqiaogao@163.com
}

Keywords: FE-SPH coupling method; water impact; simulation

Abstract. The water entry of torpedo based on FE-SPH coupling method was studied. Firstly, the model of water entry of high-speed torpedo is established. In the case of grid structure, the numerical simulations verify the effectiveness of the model by comparing with the experimental results. Then the impact forces of the water entry about the torpedo were calculated with different entry angles and entry velocities. The results were compared with the results of FE method. The conclusions are expected to be beneficial to forecast the impact forces of water entry about the torpedo based on FE-SPH coupling method.

\section{Introduction}

SPH (Smooth Particle Hydrodynamic) is one of the meshless Lagrangian method and has distinctive characteristic in solving the impact problems. In this method the particles were used to represent the specific physical parameters during the process of solving the impact problem. So the interface between the different materials can be identified clarity through the motion of the whole particles while the interaction between the particles can be handled properly. Since there is no grid in the computational region, so the simulation problem caused by large deformation disappeared. As the particles have the Lagrangian property that the particles can track the movement of material, record the deformation history of material and the change of interface. So the SPH method can be used in the high speed collisions, especially hypervelocity collision that finite element method or finite difference method could not be used situation. However, the SPH method need to determine the particles in each calculation domain, and the search algorithm will cost huge computational time, so the computational efficiency is significantly lower than the finite element method(FEM).[1,2]

For the water entry problem of torpedo, the large deformation during the impact process usually occurs only in a localized area of impact. In order to improve the simulation accuracy, the large deformation problem muse be solved. The couple of SPH method and FE method is one of the ways to solve the problem. In the large deformation the finite elements can convert to smooth particles automatically based on the coupled algorithm. The large deformation, broken and scattered problem can be solved by smooth particles. While the finite elements were still used in other zones.[3,4]

Currently, most of the researchers only studied the local model of torpedo. While the whole torpedo modeling, and the use of fluid-structure coupling algorithm will be closer to the actual situation, there is more information could be obtained. In this paper, the whole torpedo model was established by finite element method and the water was modeled by smoothed particle hydrodynamics method. The whole simulation model was combined by FE-SPH couple algorithm. Based on the model, the effect of water entry angle and water entry velocity on the impact forces of the torpedo during the water entry process was studied.

\section{SPH Method in Fluid-Structure Interaction}

\section{The discrete of control equations}

The SPH method used in this paper was first proposed by Monaghan[5] and was widely used in various fields. Its core is an interpolation theory. One of the variables $f(x)$ which may represent 
density, pressure, speed and so on. The integrate of $f(x)$ taken on the entire region, and its form can be shown:

$$
\langle f(x)\rangle=\int_{\Omega} f\left(x^{\prime}\right) W\left(x-x^{\prime}, h\right) d x^{\prime}
$$

In the Eq. (1), $\langle f(x)\rangle$ is the approximate kernel of $f(x), W$ is smooth kernel function and is the functions about the distance between $(x-x)$ two particles and smoothing length $h$.

According to the approximation process of SPH method and combined with the differentiable nature of nuclear, and the variables $f(x)$ were substituted by density $\rho$, speed $v$, pressure $P$ and internal energy $e$. Then the Navier-Stokes equations can show in the particles discrete form (not considering viscosity)[6]:

$$
\begin{aligned}
\frac{d \rho_{i}}{d t} & =\sum_{j=1}^{N} m_{j} v_{i j}^{\beta} \frac{\partial W_{i j}}{\partial x_{i}^{\beta}} \\
\frac{d v_{i}^{\alpha}}{d t} & =-\sum_{j=1}^{N} m_{j}\left(\frac{P_{i}}{\rho_{i}^{2}}+\frac{P_{j}}{\rho_{j}^{2}}+\prod_{i j}\right) \frac{\partial W_{i j}}{\partial x_{i}^{\alpha}} \\
\frac{d e_{i}}{d t} & =\frac{1}{2} \sum_{j=1}^{N} m_{j}\left(\frac{P_{i}}{\rho_{i}^{2}}+\frac{P_{j}}{\rho_{j}^{2}}+\prod_{i j}\right) v_{i j}^{\beta} \frac{\partial W_{i j}}{\partial x_{i}^{\beta}}
\end{aligned}
$$

In many applications of SPH method, researchers often do not use the continuity equation (2) to approximate density, but direct use of density approximation summation, namely

$$
\rho_{i}=\sum_{j=1}^{N} m_{j} W_{i j}
$$

\section{Fluid-structure coupling model}

The fluid-structure coupling model used in the paper was shown in the Fig. 1. It can be seen in the figure that there is no transition units on the fluid-structure coupling surfaces. The SPH domain and FE domain separated completely, and connected by a contact surface.

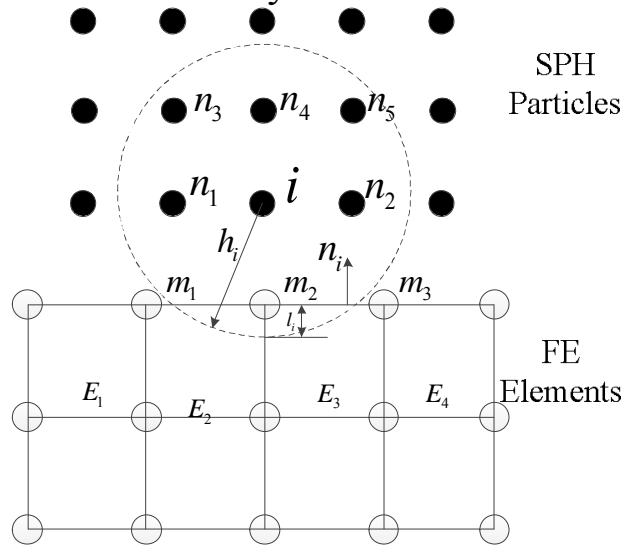

Fig. 1 interface model of SPH and FE particles

The Leap-Frog mode was used to discrete the N-S equations in the SPH, while the central difference scheme was used to solve the display kinetic equations in FE. The interaction between SPH particles and FE elements was calculated by the contact algorithm. When there is no contact between the particles and finite elements, FE unit had no effect on the calculation of SPH particles. When the SPH particles and FE units were in contact during the simulation, and the SPH particles had the trend of penetrating the surface of FE units, FE elements or nodes are not directly involved in the SPH particles approximate calculation. In this case, a contact force between the SPH and FE unit was introduced to solve this problem based on the penetration depth. The force was calculated in the SPH approximate simulation as an external force. The function of the force can be explained that there was an adjustable spring stiffness between the SPH particles and the FE units. And it can limit the penetration between the particles and FE units. 
Since the effect of FE units acted on the SPH particles through the interfacial contact force. The calculation of SPH particles and FE units is independent, so the contact coupling numerical algorithm implementation was very easy, and the interface contact force can also vary with the actual situation. The EBE (Element-By-Element) algorithm was used in the simulation. In order to reduce the amount of calculation and matrix inversion at each time step, the calculation of finite element overall stiffness was changed into single matrix-vector product. The smooth glossy length lengths method was used in the SPH algorithm to avoid the impact.

\section{Simulation Model}

The simulation model used in the paper is the torpedo MK-46[7]. The diameter is $324 \mathrm{~mm}$, the total length is $2790 \mathrm{~mm}$, and the mass is $215 \mathrm{~kg}$. The shell thickness of model is $10 \mathrm{~mm}$ and the material is steel. The density of steel is $7840 \mathrm{~kg} / \mathrm{mm}^{\wedge} 3$, the Young's modulus is $210 \mathrm{Gpa}$, the Poisson's ratio is 0.3 and the yield strength is $235 \mathrm{Mpa}$. The three dimensional model was completed in the UG software, and the meshing and material definition was done in the ANSYS software. The grid of torpedo was shown in the Fig. 2.

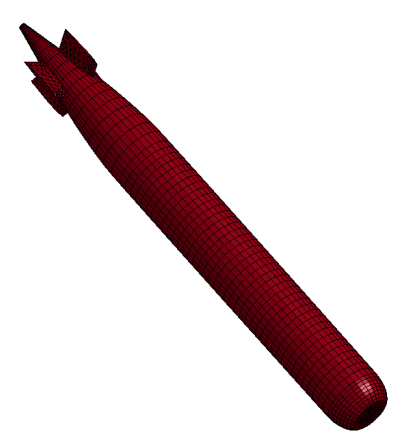

Fig. 2 Grid of torpedo

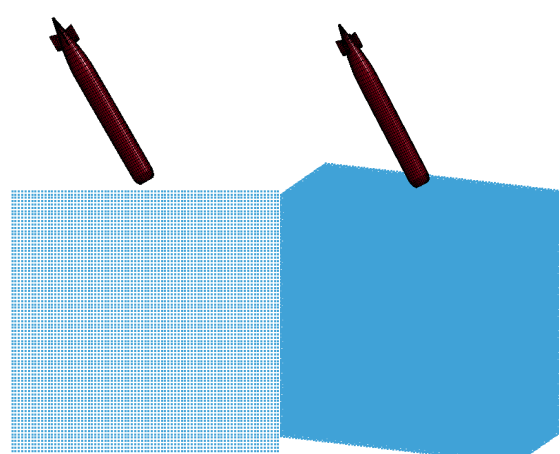

Fig. 3 The model of water entry of torpedo $\left(45^{\circ}\right.$ entry angle)

The fluid part was modeling in SPH method and there was no consider the effect of waves on the water entry process. The null material model and state equations were used to describe the water. In the LS-DYNA software the Gruneisen equation was used to simulate the water.[8]

Simulation model was shown in the Fig. 3 . The size of water domain is $2 \times 1 \times 0.5$ meters, and there are 45000 fluid particles and 4632 boundary nodes. The dynamic boundary condition was used in the simulation. Three virtual particles were set in and out the wall. The position of these particles was still and the velocity was zero.

\section{Simulation Results}

\section{Process Analysis of water entry}

Fig. 4 was the water entry process of torpedo. The water entry angle was 45 degree and the velocity was $50 \mathrm{~m} / \mathrm{s}$.

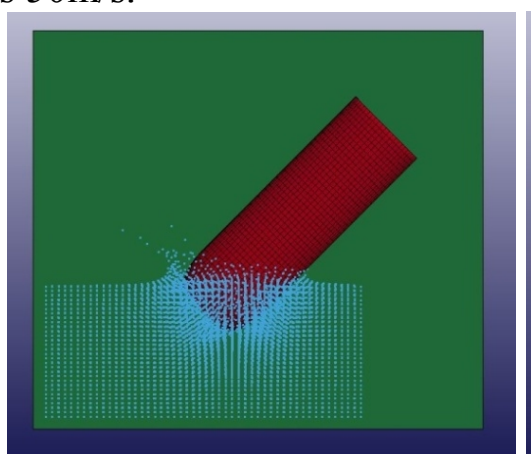

$\mathrm{t}=4.5 \mathrm{~ms}$

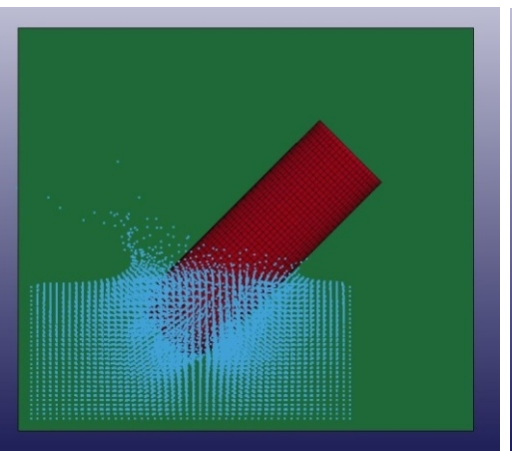

$\mathrm{t}=12.4 \mathrm{~ms}$

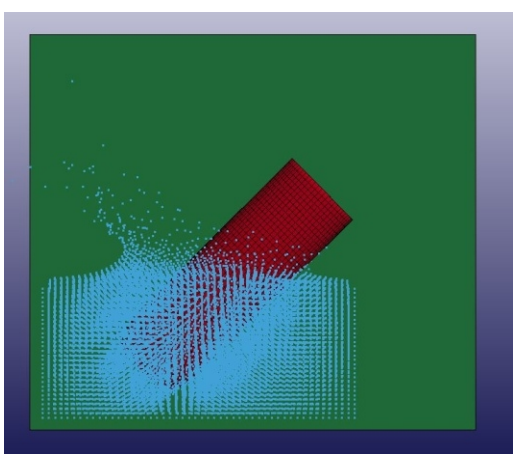

$\mathrm{t}=19.7 \mathrm{~ms}$

Fig. 4 The water entry of torpedo 
From the result it shown that the SPH-FE method can not only simulate the general case of water entry stably but also can simulate the spatial details clearly at different time. Compared with the classical FE method, the SPH-FE algorithm changed the large deformation grid into the smooth particles, which can avoid the distortion of mesh. So the rising of liquid level and the splashing of spray was simulated well. However the computational time cost had increased.

Fig. 8 was the pressure contour of the torpedo at the different water entry time.

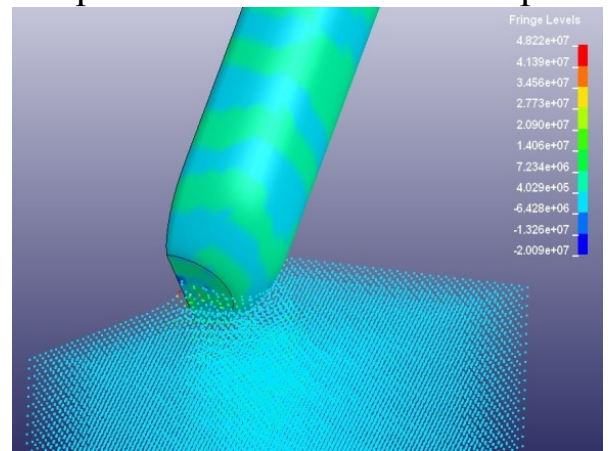

$\mathrm{t}=2.5 \mathrm{~ms}$

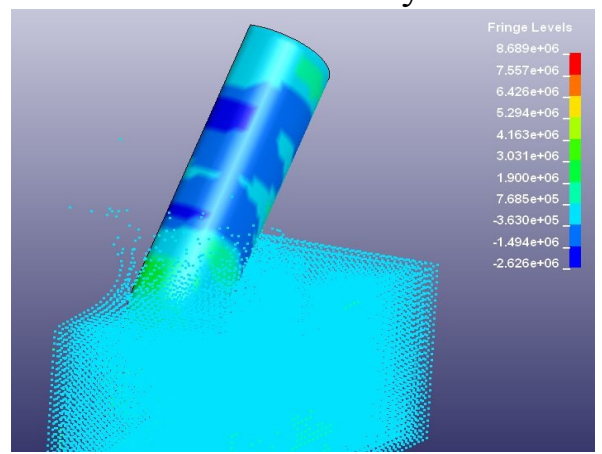

$\mathrm{t}=8.3 \mathrm{~ms}$

Fig. 5 The pressure contour of torpedo

From the Fig. 5, it can be seen that the FE-SPH method can describe the force changes during the water entry process. At the early stage of water entry, the torpedo will suffer a great impact pressure and the pressure value is about $85.2 \mathrm{MPa}$. The value is much less than the yield stress of torpedo head shell, so that the pressure on torpedo shells couldn't make the shell plastic deformation or even destruction. As the body continues to go into the water, the structure response was expansion. And the stress spread gradually from the head to the tail in the form of waves. Pressure value in the tail in much less than the head and the impact load act on the structure became stable gradually. The magnitude of the impact load would become smaller but last longer.

At the same time it can be concluded that the slamming pressure distribution on the head of torpedo was uneven. The largest location of the pressure was the portion of contact with water. Pressure acted on the torpedo passed from the head to tail. Regardless of the vertical water entry or water entry with an angle, the torpedo head was the main energy-absorbing member. So in the design of torpedo head shape should choose smaller water contact shape or install a buffer headgear ways to reduce the impact load, protecting the components in the head.

\section{Effect of water entry velocity}

For the further analysis of water entry process, under the water entry angle was 45 degrees case, the water entry velocity changed from $40 \mathrm{~m} / \mathrm{s}$ to $60 \mathrm{~m} / \mathrm{s}$. The simulation results shown in the Fig. 6 .

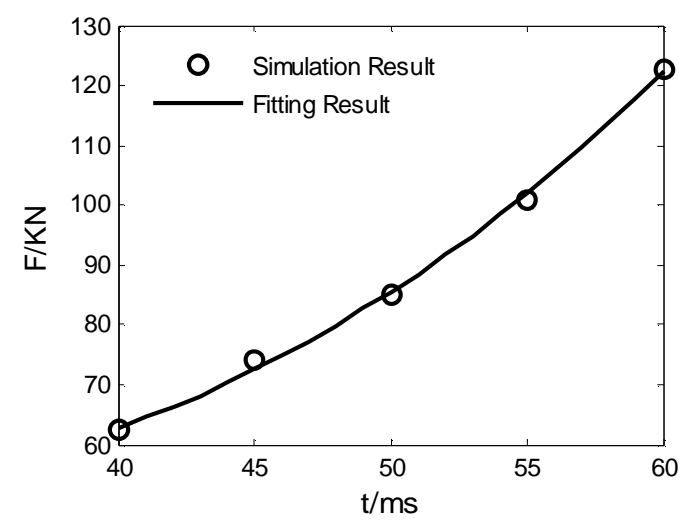

Fig. 6 Impact force of different water-entry velocities

The quadratic curve fitting functions from the results can be written as:

$f(x)=0.07057 x^{2}-4.095 x+113.8$ 
From the simulation results can be drawn that, the water entry velocity was larger and the impact load peak was larger, while the peak time was shorter. So reduced the water entry velocity will be very effective in reducing the shock suffered by torpedo.

\section{Effect of water entry angle}

Studied the impact force results that the water entry velocity was $50 \mathrm{~m} / \mathrm{s}$, while the water entry angle changed from 30 degrees to 90 degrees. Table 1 were the axial forces and normal forces results at different water entry angles.

Table 1 The max impact forces of different water-entry angles

\begin{tabular}{|cccccc|}
\hline Angle of Water entry $\left[^{\circ}\right]$ & 30 & 45 & 60 & 75 & 90 \\
\hline Axial Force[KN] & 26.9 & 40.2 & 42.4 & 44.2 & 72.20 \\
\hline Normal Force[KN] & 28.8 & 35.2 & 16.7 & 8.9 & 0 \\
\hline
\end{tabular}

From the table 1, it can be seen that the impact force increased along with the water entry angle. Especially when the angle was 90 degrees the axial force reached to the maximum, indicating that this case was most unsafe situation. While the normal force increased with the water entry angle at primary then decreased with water entry angle.

\section{Conclusions}

The water entry impact force and pressure of torpedo at different water entry angles and velocities were studied based on the FE-SPH coupling algorithm, and draw the following conclusions:

(1) During the water entry process, the torpedo will suffer high impact loads, which will affect the structure sharply. Therefore, in order to ensure the safety and reliability of the water entry process, it is necessary to reinforce the strength near the head of the torpedo.

(2) The impact loads of the torpedo increased with increased of water entry velocity. Minimize the water entry velocity is very essential.

(3) The axial forces increased with water entry angles while the normal force decreased along with the angle. The most dangerous situation is when the angle reached 90 degrees.

\section{References}

[1] Lucy L R, A numerical approach to the testing of the fission hypothesis, The Astron J, 82 (1977) 1013-1024.

[2] Monaghan J J, Smoothed particle hydrodynamics, Rep Prog Phys, 68 (2005) 1703-1759.

[3] Sun Zhanhua, Dynamics response analysis of penetration based on FE-SPH adaptive coupling method, Hunan University, 2012. (In Chinese)

[4] Wang Ji, Linking of Smoothed Particle Hydrodynamics Methods to standard finite element methods and its Application in Impact Dynamics, Department of Modern Mechanics University of Science and Technology of China, 2006. (In Chinese)

[5] Monagham J J., An introduction to SPH, Computer Physics Communications, 48 (1988) 89-96.

[6] Xu Qingxin, Study of Some Impact Dynamics Problems Based on Smoothed Particle Hydrodynamics Method, Shanghai Jiao Tong University, 2009. (In Chinese)

[7] Pan Guang, Du Xiaoxu, Song Baowei etal. Toeprdo Mechanics, Shaanxi Xi'AN, 2013.

[8] He Haoshan, Hu Renxi, etal, ANSYS12.0LS-DYNA User Manual, Beijing ,2011. (In Chinese). 